\title{
Approximate Contagion Model of Common Knowledge on Facebook
}

\author{
Gizem Korkmaz \\ Virginia Tech \\ gkorkmaz@vbi.vt.edu \\ S. S. Ravi \\ University at Albany-SUNY \\ sravi@albany.edu
}

\author{
Chris J. Kuhlman \\ Virginia Tech \\ ckuhlman@vbi.vt.edu \\ Fernando Vega-Redondo \\ Bocconi University \\ fernando.vega@unibocconi.it
}

\begin{abstract}
Computational modeling of information exchange over social media is important for understanding coordination in online environments. Many contagion dynamics models that have been used to model Twitter, Facebook, and blog information transmission are polynomial-time computable, and hence can be efficiently simulated on networked populations. Game-theoretic models of collective action (i.e., coordination problems that require common knowledge among agents), however, have dynamics that are controlled in part by specific network structures such as cliques and bicliques. Contagion dynamics with these models cannot be efficiently computed because finding all bicliques in a graph, for example, is an NP-hard problem. We investigate a recent model of common knowledge dynamics that represents information spread on Facebook - in which the biclique is the characterizing structure - and convert the model into an efficiently computable one by using an approximation. We demonstrate this through experiments on seven different graphs for a total of 168 sets of conditions, including a 4-order of magnitude span in dynamics parameter values. Our approach speeds computations in two ways: $(i)$ it obviates the need to find all bicliques in a social network, which is a very timeconsuming task (computations can take 30 to 120 hours or more of wall clock time), and (ii) it reduces the time of simulation computations, in some cases by well over an order of magnitude. Our method also enables evaluation of much larger networks that are being mined from social media.
\end{abstract}

\section{Categories and Subject Descriptors}

I.6.4 [Simulation and Modeling]: Model Validation and Analysis; I.6.3 [Simulation and Modeling]: Applications; J.4 [Social and Behavioral Sciences]: Sociology

Permission to make digital or hard copies of all or part of this work for personal or classroom use is granted without fee provided that copies are not made or distributed for profit or commercial advantage and that copies bear this notice and the full citation on the first page. Copyrights for components of this work owned by others than ACM must be honored. Abstracting with credit is permitted. To copy otherwise, or republish, to post on servers or to redistribute to lists, requires prior specific permission and/or a fee. Request permissions from permissions@ acm.org.

HT '16, July 10-13, 2016, Halifax, NS, Canada

(C) 2016 ACM. ISBN 978-1-4503-4247-6/16/07 . \$ $\$ 15.00$

DOI: http://dx.doi.org/10.1145/2914586.2914630

\section{Keywords}

contagion processes, modeling, simulation, common knowledge, coordination, approximate models

\section{INTRODUCTION}

\subsection{Background, Motivation, Related Work}

Computational modeling of information exchange over social media is important for understanding online coordination. The existing models that study information diffusion on (online) social networks of agents (vertices) mainly focus on 2-state contagion dynamics, where state 0 (resp., 1) means an agent does not (resp., does) possess a contagion. Some examples of contagions are information, rumors [12], fear [7], and social unrest [8]. A small sample of these models includes threshold models [9, 4, 23], independent cascade and linear threshold models [13], stochastic models [18], and others $[12,19]$ to model information spread on Twitter [18, 17, 8], Facebook [20], and blogs [10]. Another class of models is SIR- (susceptible-infectious-recovered) and SIS-based models, including multi-contagion variants that have been used for generalized contagions [6] and web browser use [3]. All of these models are pure influence models in the sense that an agent in state 0 changes to state 1 based on the number, $\theta$, of its neighbors that are already in state $1(\theta$ is an agent's threshold). Moreover, the models are efficiently computable so that simulations of contagion spread using these models can be performed in polynomial time; e.g., [15].

Another class of contagion studies is based on dynamic game-theoretic models of collective action (e.g., Chwe [5], Korkmaz et al. [14]). These are coordination problems, in which each agent makes a decision, with the potential to achieve mutual benefits only if her decision is consistent with those of others. Based on these models, agents in state 0 may change state simultaneously when none of them are currently in state 1 . This collective behavior requires that not only agents know about each other (e.g., their thresholds) but also this information is common knowledge (CK). The contagion dynamics in these models depend on specific network structures such as cliques [5].

In this paper, we focus on the model proposed in [14], herein called CKF, for collective action through Common $K$ nowledge on $F$ acebook. Among other mechanisms, it models the unique aspect of Facebook communication where users can post information on their own or their friends' walls (or timelines). For example, if agent $v_{a}$ posts a mes- 
sage on agent $v_{b}$ 's timeline, then $v_{b}$ 's friend $v_{c}$ can also read the post. Hence, this model captures friend-of-friend communication. (The relevant parts of the model are overviewed in Section 2).

It is demonstrated in [14] that the characterizing network structure for achieving CK among a set of agents in a communication network is the biclique (a complete bipartite graph). The problem is that computing all vertex-maximal bicliques in a network is an NP-hard problem [1]. An important practical result is that identifying all bicliques in a network using a serial code can take a wall clock time of 120 hours or more on high performance computing nodes (Intel Sandy Bridge processors), even for networks with a few tens of thousands of vertices. This is a required step for computing contagion dynamics on networks, meaning that computing network dynamics with the CKF model is NP-hard. The upshot is that contagion spreading can be studied only on relatively small networks, while graph-based data sets with a million vertices are becoming more common [17].

In this paper, we present an approach that retains only one particular type of biclique, resulting in polynomial time computations for network dynamics and obviates the need for preprocessing computations of bicliques.

\subsection{Contributions of This Work}

First, our proposed solution is to utilize only those bicliques that form star subgraphs, which are efficiently computed, and use these as CK sets. Hence, we ignore a large number of bicliques in a network (as will be seen). Second, we identify a stylized class of networks, with particular structures, for which our approach does not perform well in computing contagion dynamics in the worst case. We also present data on bicliques illustrating that our approximation ignores hundreds of thousands of bicliques in some cases, so these CK sets are ignored in dynamics computations. The point is that it is not obvious a priori that our approach will be effective. Third, we provide simulation data for contagion dynamics on seven networks, ranging from mined social network data to stylized networks, demonstrating that our approximate approach yields very good results on practical networks. These networks have wide ranges in properties (e.g., the numbers of bicliques in graphs range over 5 orders of magnitude and thresholds for the study of dynamics range over 4 orders of magnitude, among other variables). Fourth, we demonstrate the time savings in computing bicliques (which can be over 100 hours) and in computing network contagion dynamics through simulation (where we demonstrate a speedup of a factor of 50 or 70 in some cases). Fifth, our approach provides a way to study larger networks in practice.

We emphasize that our focus here is primarily on social network structures, which are very often "heavy-tailed" in degree distribution. That is, social networks often have a smaller group of vertices with high connectivity, similar to those with scale-free or power law degree distributions.

\section{CKF CONTAGION MODEL AND EXAM- PLE DYNAMICS}

The model of common knowledge on Facebook (CKF) considers agents who have private information about their information triple $\left(\mathrm{ID}_{i}, x_{i}, \theta_{i}\right)$. Here, $\mathrm{ID}_{i} \in \mathbb{N}$ is the identifier, $x_{i} \in\{0,1\}$ is the state, and $\theta_{i} \in \mathbb{N}$ is the threshold of agent $v_{i}$. The interaction between the agents is represented by a communication network in which a link between two agents means that they share their ID's, states and thresholds on each others' wall/timeline. This information is also observable by friends-of-friends. Agents who are connected such that they form a biclique (additional edges may be present among agents and have no effect) know their own information triple $\left(\mathrm{ID}_{i}, x_{i}, \theta_{i}\right)$, and those of every other agent in the biclique. Further, each agent $v_{i}$ knows that the other agents know $v_{i}$ 's triple, and so on [14]. This is the common knowledge, which facilitates coordination among agents, producing collective state changes and contagion spread [14, 21].

We address the computation of contagion spread that is governed by biclique subgraphs in networks, because this is the network structure that gives rise to common knowledge [14]. Three $K_{4,4}$-bicliques with different threshold assignments (listed next to the vertices) are given in Figure 1 to illustrate contagion dynamics. Each of these networks represents a set of 8 agents (vertices) who share common knowledge of ID's, states, and thresholds. That is, each vertex $v_{j} \in V\left[K_{4,4}\right]$ knows $\left(v_{i}, x_{i}, \theta_{i}\right)$ for all $v_{i} \in V\left[K_{4,4}\right]$, where $V\left[K_{4,4}\right]$ is the set of vertices in a $K_{4,4}$ biclique. We assume that all vertices $v_{j}$ in each example are originally in state 0 ; i.e., $x_{j}=0 \forall v_{j}$, the inactive state.

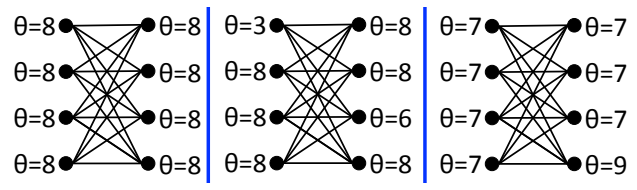

Figure 1: Three $K_{4,4}$-bicliques with 8 agents. Each of the three bicliques has threshold assignments to vertices as shown. In the left and center bicliques, all vertices change state from 0 to 1 . In the right $K_{4,4}$, the 7 vertices with $\theta=7$ change to state 1 , but the lower right vertex with $\theta=9$ remains in state 0 .

In the first (left) example, each vertex has threshold 8, meaning each vertex requires 8 vertices to be in state 1 (counting itself), in order to change to state 1. Since each vertex knows the states and thresholds of all other vertices in the biclique, and each vertex $v_{j}$ knows that all other vertices know that $v_{j}$ knows their states, and so, recursively, each $v_{j}$ reasons that all vertices have their threshold condition satisfied and will change to 1 . Hence, all 8 vertices change state, even though none of the vertices were originally in state 1 . This is how contagion can result from knowledge and coordination among vertices that share common knowledge.

The middle $K_{4,4}$ of Figure 1 has the difference that now two vertices have thresholds $\theta<8$. Since there are 8 vertices sharing common knowledge, the same logic as above applies, and all 8 vertices will transition from state 0 to 1 simultaneously.

The last (right) $K_{4,4}$ has one vertex with $\theta=9$; the remaining vertices have $\theta=7$. The vertex with $\theta=9$ will not change state to 1 because it requires a total of nine vertices, but there are only eight in this common knowledge set. However, the remaining seven vertices have $\theta=7$ and hence will transition to state 1 , owing to common knowledge. Thus, this example shows that within a CK set, a subset of vertices may change state.

Another parameter in the model is the participation probability, $p_{p}$, which is the probability that an agent will be 
online and participating in the contagion process at each time step. If an agent is not participating, it does not contribute to the contagion dynamics (so that its data triple is not used), but since the person's Facebook wall still exists, the node and its incident edges are still part of CK sets.

Note that the CKF model does not require seed nodes (initially assigned state 1) in order for contagion diffusion to take place, unlike many other models $[9,4,23,13,18]$ (unless $\theta=0$ in these other models).

Bicliques play an important role in the dynamics of the model and they are the source of the computational issue. Hence we focus on this aspect of it. Other aspects of the model can be found in [14].

\section{RESULTS: APPROXIMATE COMMON KNOWLEDGE MODEL OF FACEBOOK}

The CKF model is not efficiently implementable because it requires identification of all node-maximal bicliques (CK sets) in a graph, which is an NP-hard problem [1]. Here we propose an approach to make the model efficiently implementable by considering only those bicliques that are stars as an approximation to capture the dynamics of the CKF model. We are motivated here by social networks, which typically possess scale-free or exponential degree distributions, and hence there are nodes in these graphs with high degree and, consequently star-shaped bicliques with many nodes. However, the approximate model works with other network structures, as we show below. We use the following definitions throughout:

- Star biclique: A biclique in which one bipartite set has one vertex. Each node in the graph with $n$ vertices is the center vertex of a star, and hence there are $n$ stars.

- Non-star biclique: A biclique in which the size of each partite set is at least 2. These are node-maximal distinct bicliques. An example is $K_{4,4}$ in Figure 1.

First, we address the impact of computing all (both star and non-star) bicliques in a network. Then a toy network example is provided to illustrate that the proposed approximation can be poor in generating the same results as the full model, in the worst case. Next we provide evidence from structural analysis of the networks that indicates that our approach ignores many bicliques of appreciable size. Finally, we provide simulation data in comparing the full and approximate CKF models for a wide range of networks and structures, including over 4 orders of magnitude in thresholds, and 12 participation probabilities. These data illustrate that the approximate model, in fact, captures the dynamics of the full model very well. We also quantify the significant time savings that the approximate model provides.

\subsection{Illustrations of Computational Impacts}

Non-star bicliques were computed on several networks, some of which are used herein. Other networks (such as ca-hepth from the SNAP library [16]) could not be used because enumerating non-star bicliques were stopped after 120 hours of compute time. Computing these bicliques on the Enron network of Table 1 required 62 hours using a serial algorithm. These computations were run on dual-socket Intel Sandy Bridge (Dell C6220) E5-2670 2.60GHz 8-core processors with 64GB 1600MHz DDR3 RAM.

\subsection{An Example Illustrating Worst Case Per- formance of the Approximate Model}

Consider the graph in Figure 2, which is a collection of eight $K_{4,4}$ subgraphs connected in a circle configuration. If all vertices have threshold 8 , then since each $K_{4,4}$-biclique has 8 vertices, the nodes in each biclique will change to state 1 in one time step. However, if instead only stars are considered as CK sets, then there will be no diffusion since the maximum sized star has 6 vertices. Hence, the approximate (star biclique) model can be arbitrarily poor in computing the number of nodes in state 1 . We note that this example requires a particular structure (collections of subgraphs that are precisely bicliques with no additional edges) that is not often representative of social networks (as described above), and a particular threshold.

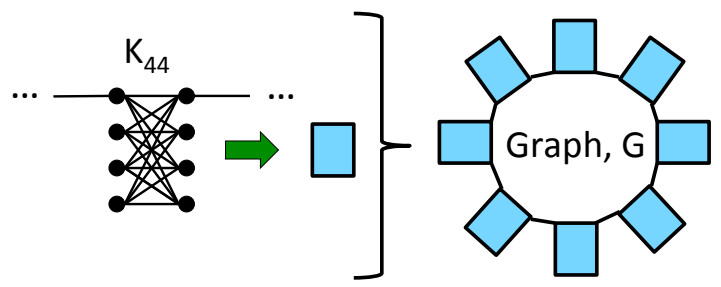

Figure 2: Graph of 8 connected $K_{4,4}$-bicliques in a ring fashion. If all vertices have threshold $\theta=8$, then the full CKF model produces a cascade, but the reduced model using only star bicliques produces no diffusion.

\subsection{Data Illustrating the Number of Bicliques Ignored in the Approximate Model}

Table 1 contains the networks of this study, separated into real (i.e., mined) and scale-free (SF) stylized networks. FB is a Facebook user network [22], $\mathrm{AH}$ is a school of youth interactions [11], P2PG is a peer-to-peer network, and Enron is an Enron email network [16]. The remaining (SF) networks were generated by a standard preferential attachment method [2].

Table 1: Characteristics of social networks analyzed. Here, $n$ and $m$ are the numbers of vertices and edges, respectively; $d_{a v e}$ and $d_{\max }$ are the average and maximum degrees; $n_{b c}$ is the number of non-star bicliques, and $n_{C K}$ is the number of distinct CK sets generated on these bicliques, respectively.

\begin{tabular}{lrrrrrr}
\hline \hline Net & \multicolumn{1}{c}{$n$} & \multicolumn{1}{c}{$m$} & $d_{\text {ave }}$ & $d_{\max }$ & \multicolumn{1}{c}{$n_{b c}$} & $n_{C K}$ \\
\hline FB & 43,953 & 182,384 & 8.30 & 223 & 614,504 & 258,668 \\
\hline AH & 2,448 & 5,277 & 4.31 & 10 & 10,388 & 1,496 \\
\hline P2PG & 10,876 & 39,940 & 7.34 & 101 & 11,332 & 5,663 \\
\hline Enron & 33,696 & 180,811 & 10.7 & 1,383 & $789,099,550$ & $50,467,699$ \\
\hline \hline SF-1 & 4,956 & 45,031 & 18.2 & 269 & $84,599,732$ & $41,695,882$ \\
\hline SF-2 & 4,974 & 44,886 & 18.0 & 262 & $99,054,962$ & $48,734,344$ \\
\hline SF-3 & 4,941 & 44,973 & 18.2 & 263 & $130,892,216$ & $64,363,550$ \\
\hline \hline
\end{tabular}

The number of non-star bicliques, $n_{b c}$, is greater than the number of distinct maximal CK sets based on the non-star bicliques, $n_{C K}$. For example, ten vertices may form the following four non-star bicliques: $K_{2,8}, K_{3,7}, K_{4,6}, K_{5,5}$. In fact, there are $\sum_{j=2}^{5}\left(\begin{array}{c}10 \\ j\end{array}\right)=627$ distinct non-star bicliques on 10 vertices. However, each of these bicliques reduces to a single CK set that is composed of the same set of ten vertices. 
We have $n_{C K} \gg n$ for most of the networks, indicating that there are many more non-star bicliques than the number of star bicliques, $n$, in the graph. Hence the number of bicliques that we ignore in the approximate model is large. Next, we look at the sizes of these bicliques.

Figure 3 shows how the frequencies of CK set sizes vary when generated from star and non-star bicliques. Hence, the full model utilizes all of these bicliques. In these plots, we observe that there are many non-star bicliques of large sizes, and hence it is not clear that ignoring these bicliques will preserve the contagion dynamics of the full model. In particular, note that in Figure 3(a), there are $10^{3}$ to $10^{7}$ bicliques with sizes ranging from 10 to 70 , which are being ignored in the approximate (star biclique) model. Similar observations are relevant for at least the Enron and FB networks of Figure 3(b).

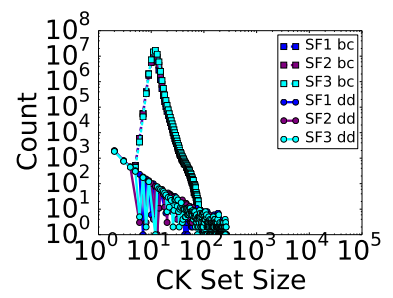

(a)

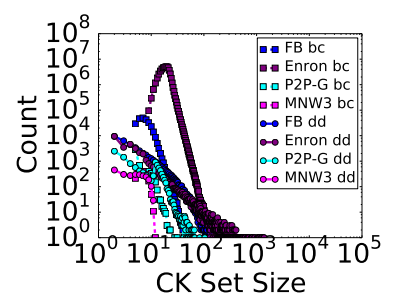

(b)
Figure 3: Frequency of common knowledge (CK) set sizes based on $(d d)$-star bicliques (circles), and $(b c)$ non-star bicliques (squares). (a) Three SF graphs. (b) Four real networks.

\subsection{Experimental Comparison Of Approximate and Full Common Knowledge Models of Facebook}

We exercise the two models - the approximate (star biclique) model and the full model - for all of the networks in Table 1 . The only difference between the two models is the approximation by including only star bicliques in the approximate model, versus all bicliques in the full model; otherwise the models are identical. For each (model, network) pair, we run one simulation (each consisting of 50 runs of the contagion process) that contains each of the $\left(\theta, p_{p}\right)$ pairs given in Table 2, for a total of 168 sets of conditions. The $p_{p}$ values differ for $\theta_{l}$ and $\theta_{h} ; \theta_{h}$ was selected because it is the maximum threshold for which diffusion will occur in the full CKF model. In the subsequent plots, each curve represents the time-pointwise average of the contagion size over the 50 runs. The solid curves represent the full (original) model, where all CK sets from all bicliques (including stars) are utilized, and dashed curves represent the approximate model where only stars are used for CK sets. As we will see, the solid and dashed curves overlap on the plots, indicating very good agreement of results between the models. This is the case for all 168 sets of conditions; not only those plotted here.

Figure 4 contains data for the FB network. Figures 4(a) and 4(c) contain Epi curves; that is, the number of vertices newly converting to state 1 at each time, up to 30 time units. Figures 4(b) and 4(d) contain the corresponding Cum curves; that is, the cumulative number of vertices in state 1 at each time, up to 30 time units. The first two plots corre-
Table 2: Simulation matrix for each network in Table 1. Each vertex is assigned the same threshold and same participation probability in a simulation.

\begin{tabular}{ll}
\hline \hline Threshold & Participation Probabilities \\
\hline$\theta_{l}=d_{\text {ave }}$ & $0.01,0.05,0.1,0.2,0.3,0.4$ \\
\hline$\theta_{h}=d_{\max }+1$ & $0.99,0.995,0.997,0.999,0.9999,1.0$ \\
\hline \hline
\end{tabular}

spond to $\theta_{l}=9$ and the latter two correspond to $\theta_{h}=224$. For each of $\theta_{l}$ and $\theta_{h}$, the different color curves in each plot correspond to different participation probabilities, per Table 2. The highest participation probability corresponds to gray and the mimimum one corresponds to blue, for each of $\theta_{l}$ and $\theta_{h}$. Since the ordinate value on the Cum curve is the corresponding area under the Epi curve, one would expect the differences to be more gradually varying in the Cum curves. In fact, this is not the case: both sets of curves show excellent agreement between the two models, over all test parameter values for $\theta$ and $p_{p}$.

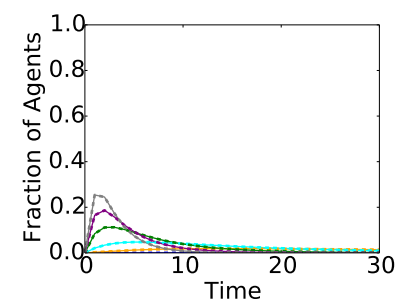

(a)

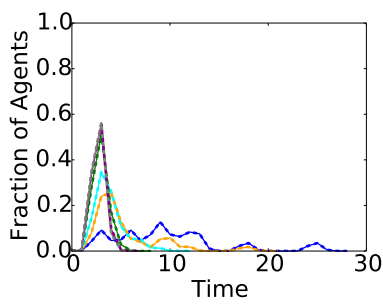

(c)

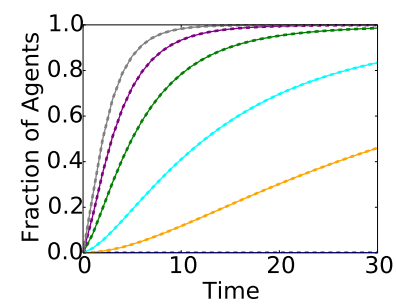

(b)

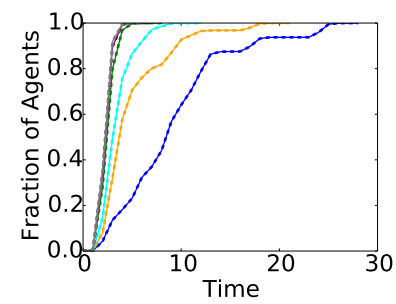

(d)
Figure 4: Fraction of agents in state 1 as a function of time for the star (dashed) and full (solid) models for network FB. (a) Epi, $\theta_{l}=9$. (b) Cum, $\theta_{l}=9$. (c) Epi, $\theta_{h}=224$. (d) Cum, $\theta_{h}=224$. The data indicate excellent agreement between the full and approximate CKF models.

Although the Epi curves are harder to match between models and hence provide a better comparison (because they are the derivatives of the Cum curves), we show the Cum curves because they are easier to inspect visually; the Epi curves for the remaining networks show the excellent agreement indicated in Figure 4.

Figure 5 shows one set of Cum curves for each of four networks: Figure 5(a) Enron, $\theta_{h}=1384$; Figure 5(b) AH, $\theta_{l}=4$; Figure 5(c) P2PG, $\theta_{h}=102$; and Figure 5(d) SF1, $\theta_{l}=18$. Note that in this plot alone, data over four orders of magnitude in threshold are presented. Again, the agreement between the full CKF model (solid curves) and the star-only biclique CKF model (dashed curves) is excellent. 
The data for Enron (Figure 5(a)) show a stair-step pattern for $p_{p}=0.999$. This is because the dynamics are very sensitive to $p_{p}$ for high thresholds. Because $\theta_{h}=d_{\max }+1$, all agents (vertices) of the subgraph centered at the vertex with degree $d_{\max }$ must be participating before vertices start to change to state 1 . This is why the $p_{p}$ values for $\theta_{h}$ are so large in Table 2.

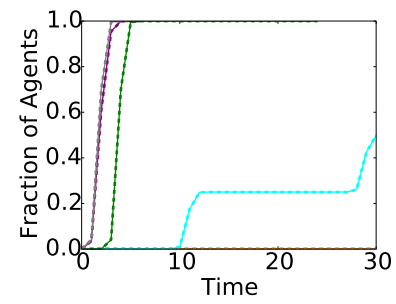

(a)

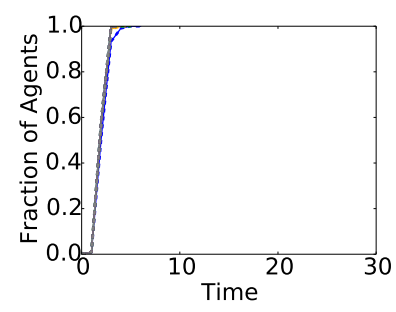

(c)

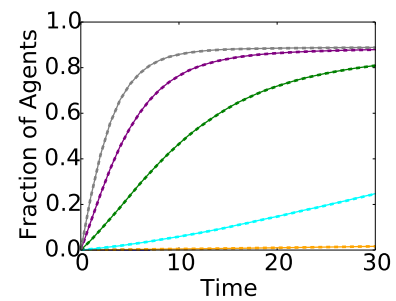

(b)

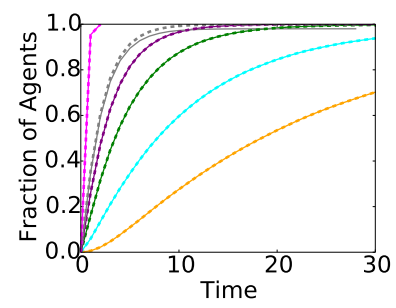

(d)
Figure 5: Fraction of agents in state 1 as a function of time for the star (dashed) and full (solid) models for different networks, thresholds, and participation probabilities. (a) Enron, $\theta_{h}=1384$. (b) AH, $\theta_{l}=4$. (c) P2PG, $\theta_{h}=102$. (d) SF1, $\theta_{l}=18$. Data over four orders of magnitude in threshold are presented. The data indicate excellent agreement between the full and approximate CKF models.

Our preliminary analysis suggests that the star-only model is a good approximation for the full model because every vertex $v_{i}$ is part of at least $d_{i}+1$ bicliques, where $d_{i}$ is the degree of $v_{i}$. That is, $v_{i}$ is a member of the CK sets that are produced from stars centered at $v_{i}$ itself and each of $v_{i}$ 's distance- 1 neighbors. It is nonetheless still surprising that the bicliques that are not stars do not alter the dynamics to some readily observable level.

\subsection{Timing Data}

The execution times were recorded for all runs of all simulations. For a specific set of conditions, a simulation of the full model takes time $t_{f u l l}$ to complete the 50 diffusion instances. The corresponding time for the star-only biclique model is $t_{\text {star }}$. The ratio $t_{\text {full }} / t_{\text {star }}$ of compute times for the full model to the approximate model were compiled and averaged. Figure 6 shows these data for each of the datadriven networks. As the number of CK sets, $n_{C K}$, increases, the ratio of computational time increases because these lists of bicliques must be traversed to determine whether vertices change state in the full model. The star-only biclique computations reduce simulation times from about a factor of 2 to a factor greater than 70 . This is on top of the time savings achieved by not having to compute all bicliques, which can be over 100 hours of wall clock time.

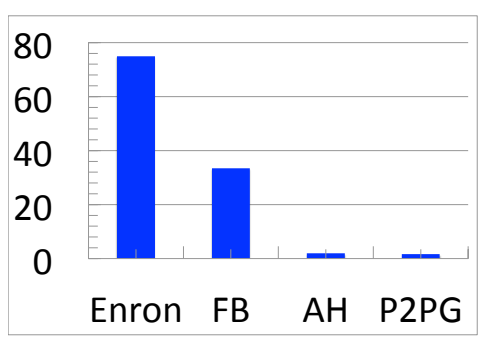

Figure 6: The average ratio of $t_{f u l l} / t_{\text {star }}$ for each of the four real networks. These data illustrate that the simulations - not counting the time to compute bicliques - with all bicliques (full model) take much longer than those that utilize star-only bicliques. Hence, the approximate model saves on computation time in addition to the time to identify bicliques.

\section{CONCLUSIONS}

Modeling and simulation of information spread on social networks enables understanding of the dynamics of coordination and collective action in online environments. Generating results in a timely fashion, in turn, requires computationally efficient implementations of model dynamics. We focus on a unique CKF model of Facebook interaction through timelines. In this model, the biclique network structure characterizes CK [14], but identifying all node-maximal bicliques - which is required for CKF contagion dynamicsis an NP-hard problem [1]. We demonstrate through experiments that it can take 120 or more hours to compute all bicliques of a network, even for relatively small graphs of tens of thousands of vertices. We introduce an approximation into the model, which is to consider only bicliques that have star structures. This makes the simulation process computationally efficient. The results from the approximate and full CKF models are in a very good agreement, thus justifying the use of the approximate model. This obviates the need to compute all bicliques, and we show that it can also reduce the time to compute contagion dynamics in simulations by up to a factor of 70 in these experiments. These results are generated on seven networks with different $n, m, d_{a v e}, d_{\max }$, and different network structures, whose numbers of biclique-based CK sets range over 5 orders of magnitude, using thresholds $\theta$ that range over 4 orders of magnitude. Future work includes using mean square error or some variant of it to quantify differences between the full and approximate model results.

\section{ACKNOWLEDGMENTS}

We thank the anonymous reviewers for their useful comments and suggestions. We thank our external collaborators and members of the Network Dynamics and Simulation Science Laboratory (NDSSL). This work was partially supported by DTRA Grant HDTRA1-11-1-0016, DTRA CNIMS Contract HDTRA1-11-D-0016-0001, and NSF NetSE Grant CNS-1011769.

\section{REFERENCES}

[1] G. Alexe, S. Alexe, Y. Crama, S. Foldes, P. L. Hammer, and B. Simeone. Consensus algorithms for 
the generation of all maximal bicliques. $D A M$, 145:11-21, 2004.

[2] A. Barabasi and R. Albert. Emergence of scaling in random networks. Nature, 286:509-512, 1999.

[3] A. Beutel, B. A. Prakash, R. Rosenfeld, and C. Faloutsos. Interacting viruses in networks: Can both survive? In Proceedings of the 18th ACM SIGKDD International Conference on Knowledge Discovery and Data Mining, pages 426-434, 2012.

[4] D. Centola and M. Macy. Complex Contagions and the Weakness of Long Ties. Am. J. Soc., 113(3):702-734, 2007.

[5] M. S.-Y. Chwe. Structure and strategy in collective action. Am. J. of Soc., 105:128-156, 1999.

[6] P. S. Dodds and D. J. Watts. A generalized model of social and biological contagion. J. of Theo. Bio., 232(4):587-604, 2005.

[7] J. M. Epstein, J. Parker, D. Cummings, and R. A. Hammond. Coupled contagion dynamics of fear and disease: Mathematical and computational explorations. PLoS ONE, 3:e3955-1-e3955-11, 2008.

[8] S. Gonzalez-Bailon, J. Borge-Holthoefer, A. Rivero, and Y. Moreno. The dynamics of protest recruitment through an online network. Nature Scientific Reports, pages $1-7,2011$.

[9] M. Granovetter. Threshold Models of Collective Behavior. Am. J. of Soc., 83(6):1420-1443, 1978.

[10] D. Gruhl, R. Guha, D. Liben-Nowell, and A. Tomkins. Information diffusion through blogspace. In Proc. of the 13th International World Wide Web Conference ( $W W W$ 2004), pages 491-501, 2004.

[11] K. M. Harris. The National Longitudinal Study of Adolescent Health (Add Health), Waves III, 2008. Carolina Population Center, UNC.

[12] K. Kawachi, M. Seki, H. Yoshida, Y. Otake, K. Warashina, and H. Ueda. A rumor transmission model with various contact interactions. Journal of Theoretical Biology, 253:55-60, 2008.
[13] D. Kempe, J. Kleinberg, and E. Tardos. Maximizing the spread of influence through a social network. In $K D D$, pages 137-146, 2003.

[14] G. Korkmaz, C. J. Kuhlman, A. Marathe, M. V. Marathe, and F. Vega-Redondo. Collective action through common knowledge using a Facebook model. In Thirteen International Conference on Autonomous Agents and Multiagent Systems (AAMAS), 2014.

[15] C. J. Kuhlman, V. A. Kumar, M. V. Marathe, S. S. Ravi, and D. J. Rosenkrantz. Inhibiting diffusion of complex contagions in social networks: Theoretical and experimental results. Journal Data Mining and Knowledge Discovery, 2014.

[16] J. Leskovec and A. Krevl. SNAP Datasets: Stanford large network dataset collection. http://snap.stanford.edu/data, Jun 2014.

[17] S. Myers and J. Leskovec. Clash of the contagions: Cooperation and competition in information diffusion. In 2012 IEEE 12th International Conference on Data Mining (ICDM), pages 539-548, 2012.

[18] D. Romero, B. Meeder, and J. Kleinberg. Differences in the mechanics of information diffusion. In $W W W$, 2011.

[19] D. Siegel. Social networks and collective action. American Journal of Political Science, 53:122-138, 2009.

[20] E. Sun, I. Rosenn, C. A. Marlow, and T. M. Lento. Gesundheit! modeling contagion through facebook news feed. In (ICWSM 2009), 2009.

[21] K. A. Thomas, P. DeScioli, O. S. Haque, and S. Pinker. The psychology of coordination and common knowledge. Journal of Personality and Social Psychology, 107:657-676, 2014.

[22] B. Viswanath, A. Mislove, M. Cha, and K. P. Gummadi. On the evolution of user interaction in Facebook. In (WOSN'09), August 2009.

[23] D. Watts. A simple model of global cascades on random networks. PNAS, 99(9):5766-5771, 2002. 